Check for updates

Cite this: RSC Adv., 2017, 7, 55830

\title{
A rapid, high yield size-selective precipitation method for generating Au nanoparticles in organic solvents with tunably monodisperse size distributions and replaceable ligands $\uparrow$
}

\author{
Steven Gravelsins and Al-Amin Dhirani (D)* \\ Size-selective precipitation (SSP) is a powerful tool for obtaining monodisperse nanoparticles. Here we \\ report a fast, high yield and tunable SSP procedure via non-solvent addition for producing nearly \\ monodisperse, organic-soluble Au nanoparticles with standard deviations as low as $\sigma<7 \%$. The addition \\ of excess ligands and judicious choice of ligand head group significantly improve both precipitate yields \\ and nanoparticle monodispersity.
}

Received 10th October 2017

Accepted 4th December 2017

rsc.li/rsc-advances

$\mathrm{Au}$ nanoparticles (NPs) possess unique size-dependent chemical and physical properties, ${ }^{1}$ and have many potential applications, including in photocatalysis, ${ }^{2,3}$ biomedicine, ${ }^{4,5}$ sensors, ${ }^{1,6}$ and materials science. ${ }^{1,3,7-9}$ Recently, there has been increasing interest in Au NPs also as nano-scale building blocks for macroscopic assemblies of materials, which have been shown to exhibit exotic behaviour. ${ }^{7,8}$ In any nanostructured material, control over the nanobuilding blocks is important as it allows for control over properties of the macroscopic material. The ability to obtain Au NPs of a desirable size with a narrow distribution allows for control not only of the size-dependent properties of the individual building blocks (e.g. surface plasmon resonance), but also over the macroscopic structure of the materials: particles with more uniform sizes are required to generate more ordered assemblies. A number of synthetic approaches have been reported to produce monodisperse (i.e. narrow size distribution) $\mathrm{Au}$ NPs in aqueous and organic media. ${ }^{10-13}$ Nevertheless, a variety of synthetic approaches (e.g. the Brust method amongst others) that employ organic solvents and generate polydisperse size distributions are widely used due to their inherent simplicity and robustness. As a result, simple methods to sort these polydisperse NPs into monodisperse fractions can be useful.

Size-selective separation or 'fractionalization' is a convenient method to achieve monodisperse NPs post-synthesis, ${ }^{\mathbf{1 4 , 1 5}}$ especially given that many synthetic methods produce NPs with polydisperse sizes..$^{14}$ There are several fractionalization techniques available, ${ }^{14,15}$ including methods such as centrifugation,

Department of Chemistry, University of Toronto, 80 St. George Street, Toronto, Ontario, M5S 3H6, Canada.E-mail: adhirani@chem.utoronto.ca

$\dagger$ Electronic supplementary information (ESI) available: Ligand exchange procedures for TEM imaging, additional histograms of nanoparticle sizes, nanoparticle film preparation. See DOI: $10.1039 / \mathrm{c} 7 \mathrm{ra11177k}$ chromatography, membrane filtration, and size-selective precipitation (SSP). The latter is based on a nanoscale phenomenon where NPs are precipitated from a solvent by adding a non-solvent or by pressurizing with gas. ${ }^{16}$ Many synthetic procedures require that NPs be purified from byproducts; as a result, precipitation-based methods are particularly convenient since such purification steps can be combined with SSP when precipitates are removed from the supernatant. ${ }^{14}$ SSP by non-solvent addition is especially appealing because of its inherent simplicity and cost-effectiveness. However, although this approach for narrowing the size distributions of NPs has been reported for semiconducting $\mathrm{NPs}^{14,17}$ and silver $\mathrm{NPs}^{18}$ in organic solvents, to our knowledge there are no such reports for gold NPs in organic solvents. We have observed that such particles have a tendency to precipitate irreversibly upon addition of a non-solvent, which may be one of the reasons for the lack of reports. Herein we report for the first time a fast, robust, high yield SSP procedure based on non-solvent addition for producing Au NPs that are organic-soluble and that exhibit tunable monodispersity. Further, we demonstrate that inclusion of excess ligands in the added non-solvent shifts the equilibrium between solubilized and precipitated NPs, affording more control over NP precipitation, and standard deviations as low as $\sigma<7 \%$ are achieved.

Starting from $\sim 6 \mathrm{~nm}+/-26 \%$ diameter Au NPs capped with tetraoctylammonium bromide (TOAB) in toluene synthesized via the widely used Brust method, ${ }^{19-21}$ our study compares various capping groups in subsequent SSP processing: TOAB, dodecylamine (DDA) and dodecanethiol (DDT). The study finds that when using ethanol (EtOH) as the non-solvent, remarkably DDA provides the best SSP yields, which is initially counter intuitive given that of the three ligand head groups studied, thiol of DDT has the strongest interaction with Au. An added feature of this result is that since DDA is relatively weakly bound 
to the NPs, the capping group can be switched in subsequent steps if desired. In addition, the present study finds that adding excess DDA to the non-solvent results in more gradual precipitation of NPs, permitting for finer size-selective separation and greater monodispersity in NP precipitates. Using the resulting SSP method, the amount of non-solvent + excess DDA added can be used to control finely both the average and standard deviation of the size distribution of the precipitated NPs. As a demonstration, we use this SSP method to controllably reduce the standard deviation of Au NP diameters ( $\sigma<10 \%$ and $7 \%$ ), and show that, with increasing monodispersity, macroscopic assemblies of resulting AuNPs exhibit significantly improved long range order.

Au NPs are first synthesized in toluene by reduction of gold chloride with sodium borohydride using a modified Brust method..$^{19}$ All glassware used in this synthesis is cleaned with piranha solution and aqua regia. TOAB is the NP ligand, and the concentration of $\mathrm{Au}$ atoms is $\sim 0.005 \mathrm{M}$. Fig. 1(a and e) show a transmission electron microscope (TEM) image of Au NPs before any SSP and a corresponding histogram of particle sizes. NPs have an average diameter of $5.8 \mathrm{~nm}+/-26 \%$.

For subsequent SSP processing TOAB ligands are replaced with DDA by adding $0.042 \mathrm{~g}$ of DDA to $5 \mathrm{~mL}$ of Brust NP solution and leaving the mixture for 30 minutes. A DDA/EtOH nonsolvent solution is prepared by adding $0.214 \mathrm{~g}$ of DDA to $10 \mathrm{~mL}$ of absolute ethanol. The DDA/EtOH solution is then added at $1 \mathrm{~mL} \mathrm{~min}^{-1}$ with a standard pipette, and the mixture is simultaneously stirred with the pipette to ensure mixing. Once a volume ratio of $5(\mathrm{~mL})$ : $5(\mathrm{~mL})$ toluene : DDA/EtOH is achieved, the NP solution is centrifuged for $15 \mathrm{~min}$ at $\sim 2400 \mathrm{rpm}$ $(\sim 900 \mathrm{~g})$. The supernatant is then transferred to another container, and the precipitate is vacuum-dried and stored. The previous steps are repeated, adding DDA/EtOH solution to the supernatant and centrifuging at toluene : DDA/EtOH volume ratios of $5(\mathrm{~mL}): 7(\mathrm{~mL})$ and $5(\mathrm{~mL}): 10(\mathrm{~mL})$, and the resulting precipitates are again dried and collected.

The precipitates are then redispersed in toluene and characterized by TEM and ultraviolet-visible spectroscopy (UV-VIS). For all TEM imaging of redispersed precipitates, DDA ligands are replaced with DDT by adding a small amount of a DDT/ toluene solution to the NP solutions to prevent NP fusion under the electron beam (see Fig. S1, ESI $\dagger$ for details). Fig. 1 shows TEM images and histograms calculated using ImageJ. The figure also shows the average diameters and standard deviations of the original Au NPs and three precipitates collected after a typical trial following the above SSP procedure. The average diameters of the precipitates collected at toluene : DDA/EtOH volume ratios of $5: 5,5: 7$, and $5: 10$ are, respectively, $6.4 \mathrm{~nm} \pm 10 \%, 5.5 \mathrm{~nm} \pm 9.9 \%$, and $5.1 \mathrm{~nm} \pm 9.3 \%$. Precipitates collected at lower DDA/EtOH portions have larger average diameters than subsequently collected precipitates, due to larger NPs having greater surface areas and thus stronger attractions with each other. As a result, larger NPs precipitate out of solution before smaller NPs. ${ }^{17,22}$ Precipitated NPs exhibit significantly narrower size distributions ( $\sim 10 \%$ for all precipitates) than the original unprocessed NPs $(d=5.8 \pm 26 \%)$, demonstrating effective fractionalization of the NPs. UV-VIS
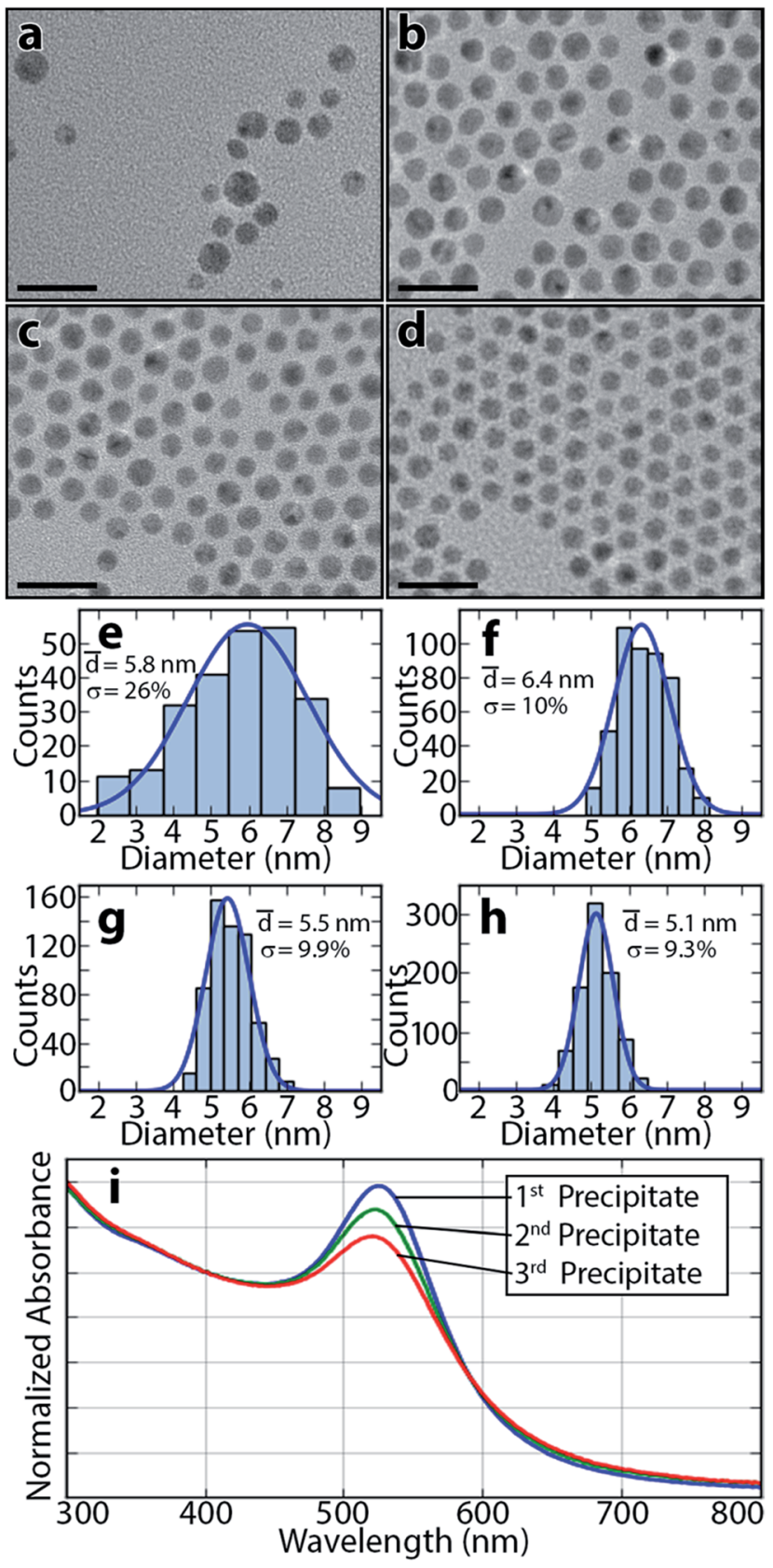

Fig. 1 (a) TEM image of Au NPs synthesized using the Brust method before SSP; (b)-(d) TEM images of 3 precipitates of Au NPs collected at toluene : DDA/EtOH ratios of $5: 5,5: 7$, and $5: 10$, respectively (DDA ligands were replaced with DDT after SSP for TEM imaging); (e)-(h) histogram and Gaussian fit of NP diameters in (a)-(d), respectively; (i) UV-VIS spectra of NP precipitates normalized at $400 \mathrm{~nm}$. Scale bars in (a)-(d) correspond to $20 \mathrm{~nm}$.

spectra of the 3 fractions (Fig. 1i), normalized to the same value at $400 \mathrm{~nm}$ to eliminate differences arising from NP concentration, exhibit increasing peaks at $\sim 520 \mathrm{~nm}$ with $\mathrm{NP}$ size. This effect is attributed to the highly size-dependent localized surface plasmon resonance (LSPR) effect., ${ }^{2,8}$

Although increasing EtOH composition is the main parameter used to separate the polydisperse NPs into more monodisperse fractions, data shown in Fig. 2 and discussed below 
show that excess ligand type (including type of ligand head group) and concentration in the non-solvent as well as temperature play important roles in tuning this SSP process. We first note that when using TOAB as the ligand and EtOH as the non-solvent, we find that the NPs precipitate irreversibly and cannot be redispersed.

For reference, Fig. 2a shows a Gaussian fit to the Brust NP size distribution shown in Fig. 1e, and Fig. 2b shows Gaussian fits as well as average particle sizes and distributions of three precipitates collected under the same conditions as in the aforementioned procedure (but from another trial than in Fig. 1 to show reproducibility). Fig. 2c displays the yields of each of these precipitates. Yields are calculated by comparing the UVVIS absorbance of the precipitates (after redispersion in toluene) with that of the Brust stock solution at $400 \mathrm{~nm}$, using that absorbance is proportional to NP concentration, and assuming negligible differences arising from NP size at this wavelength. The yields of the precipitates obtained at toluene : DDA/EtOH ratios of $5: 5,5: 7$, and $5: 10$ are, respectively,

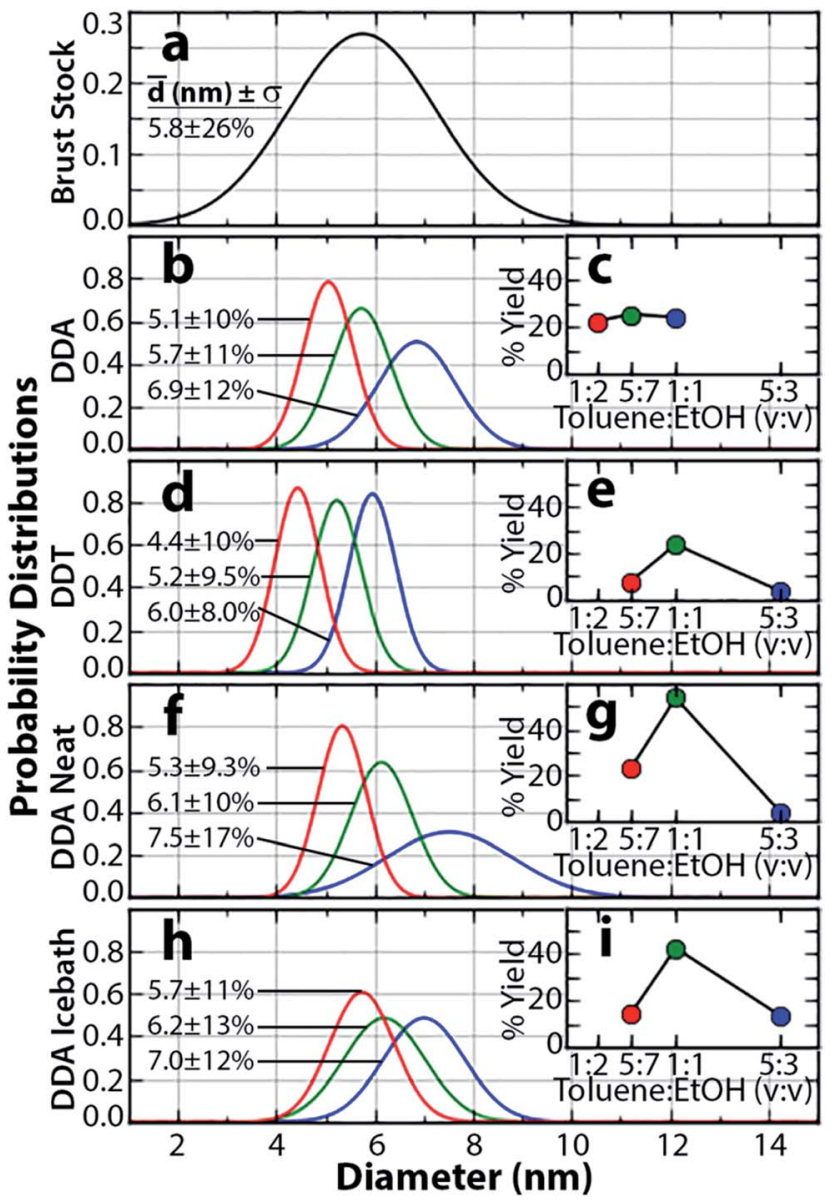

Fig. 2 (a) Gaussian fit to Brust Au NP size distribution; (b) Gaussian fits and average particle sizes of 3 precipitates collected under the same SSP conditions as in Fig. 1; (c) yields of the precipitates in (b) plotted as a function of the toluene: $\mathrm{EtOH}$ ratio at which they were collected; (d) and (e) analogous NP size and yield characterizations for SSP using DDT; (f) and (g), (h) and (i) analogous characterizations for SSP using neat $\mathrm{EtOH}$ and non-solvent addition in an icebath, respectively.
$24 \%, 25 \%$, and $22 \%$; i.e. $71 \%$ of the NPs subjected to SSP in this trial have been separated into more monodisperse fractions and recovered. Fig. 2d-e show corresponding results obtained by replacing DDA with equal molar quantities of DDT in the SSP procedure. That is, DDT is first added to $5 \mathrm{~mL}$ of the Brust NP solution to replace TOAB ligands, and the NPs are then precipitated by adding a DDT/EtOH non-solvent. Although the use of DDT in this SSP procedure also results in separation of the NPs into monodisperse fractions, the yields of the collected precipitates are substantially reduced even though the thiol head group of DDT has a stronger interaction with Au than the amine head group of DDA. Upon adding a DDT/EtOH solution to alkanethiol-capped NPs, a thin, gold-coloured film is observed on the surface of the NP solution, which we attribute to stripping of some of the DDT ligands from the NPs and the formation of gold aggregates. Ligand stripping may occur due to the polar nature of the thiol group in DDT, resulting in a higher affinity for the EtOH phase compared with the amine group in DDA. In addition, centrifugation in the DDT trials results in visible precipitates at earlier EtOH fractions compared with DDA trials; however, these precipitates in the DDT trials exhibit a higher degree of irreversible aggregation compared with those in the DDA trials. Precipitate yields obtained at toluene : DDT/ EtOH ratios of $5: 3,5: 5$, and $5: 7$ are, respectively, $4 \%, 24 \%$, and $8 \%$, indicating that only $36 \%$ of the NPs are successfully separated into monodisperse fractions and recovered. These results are particularly striking since Au NPs are readily synthesized with $\mathrm{TOAB}^{19,21}$ and $\mathrm{DDT}^{20}$ ligands. However, they may also account for the lack of reports of fractionalization of such Au NPs by non-solvent addition. In this light, substituting ligands to DDA affords a simple yet effective approach for overcoming this problem, thereby making Au NPs in organic solvents more amenable to SSP.

Further, the present study finds that the concentration of excess DDA in the added EtOH non-solvent also significantly affects the SSP process. When neat EtOH is used as a nonsolvent (i.e. no DDA is added to the $\mathrm{EtOH}$ ), higher precipitate yields are obtained at lower EtOH fractions (see Fig. 2f-g). Three precipitates obtained at toluene : EtOH volume ratios of $5: 3$, $5: 5$, and $5: 7$ have respective yields of $4 \%, 54 \%$, and $23 \%$. Thus after a toluene : EtOH ratio of $1: 1$ is achieved, $58 \%$ of the NPS have precipitated when neat EtOH is used as the non-solvent, while only $24 \%$ have precipitated when DDA/EtOH is used. We hypothesize that this shift in precipitate yields is due to decreased solubility of NPs in toluene/neat EtOH mixtures when much fewer hydrophobic DDA molecules are present. For determining NP size distributions in these trials, DDA ligands are again replaced with DDT post-SSP for TEM imaging and constructing histograms of NP diameters.

Fig. $2 \mathrm{~h}-\mathrm{i}$ shows the effect of varying temperature in this method. When DDA/EtOH solutions at $0{ }^{\circ} \mathrm{C}$ are added to the amine-capped NPs in toluene in an ice-bath, precipitate yields are again shifted towards lower DDA/EtOH fractions. This shift is attributed to NP solubility increasing with increasing temperature. Thus at lower temperatures, NPs are less soluble at a given toluene : DDA/EtOH ratio, resulting in more NPs in the precipitate phase and fewer in solution. This temperature- 
dependent equilibrium between NPs in the precipitate and solution phases has been described by theory. ${ }^{22}$

Since certain applications may require more monodisperse NPs than others, we show that tunably narrower size distributions are straightforwardly achievable with the present method by adding the non-solvent solution in more gradually increasing fractions. Fig. 3a shows a TEM image of a NP precipitate generated by increasing the toluene : DDA/EtOH volume ratio from $5: 5$ to $5: 6$ rather than to $5: 7$ used above. Fig. $3 \mathrm{~b}$ shows a corresponding histogram of particle sizes and a Gaussian fit. With the more gradual increase in non-solvent fraction, the average size of the NPs is $5.9 \mathrm{~nm} \pm 6.9 \%$, yielding a significantly smaller standard deviation compared with the $11 \%$ standard deviation obtained for the 5:7 precipitate discussed above. Although more gradual non-solvent addition should also result in narrower distributions when using neat $\mathrm{EtOH}$, results of this study indicate that more control is afforded using DDA/EtOH as the non-solvent since precipitation is shifted towards higher non-solvent fractions, resulting in a more gradual precipitation of NPs as more non-solvent solution is added (see Fig. 2(b and c) vs. Fig. $2(\mathrm{f}$ and $\mathrm{g})$ ).

NP monodispersity can be especially important when using NPs as building blocks to assemble macroscopic materials with controlled architectures. ${ }^{8}$ NP samples with various degrees of monodispersity obtained by this SSP method have been selfassembled into densely-packed monolayers to quantify the role of NP size distribution on monolayer order. A previously reported Teflon ring apparatus ${ }^{23,24}$ was used to produce NP monolayers over a $5 \mathrm{~cm}^{2}$ water surface. Films were sampled using TEM grids via the Langmuir-Schaefer technique and analysed with electron microscopy (see ESI $\uparrow$ for details). Fig. 4a displays a TEM image of a monolayer formed with polydisperse $(\sigma=26 \%)$ unprocessed NPs, and Fig. $4 \mathrm{~b}$ shows a $2 \mathrm{D}$ fast Fourier transform (FFT) of a larger $313 \mathrm{~nm} \times 313 \mathrm{~nm}$ region that includes the portion shown in Fig. 4a. Fig. 4c displays a radial average of the FFT shown in Fig. $4 \mathrm{~b}$. Analogously Fig. $4 \mathrm{~d}-\mathrm{f}$ and $\mathrm{g}-\mathrm{i}$ display TEM images and FFT characterizations of monolayers of NPs with $\sigma=10 \%$ and $\sigma=6.9 \%$, respectively. Unprocessed NPs $(\sigma=26 \%)$ assemble into monolayers with poor directional order, as is shown by the TEM image in Fig. 4a as well as the ring-like feature in the FFT in Fig. 4b. However, the FFT in Fig. 4e generated using SSP-fractionalized NPs with

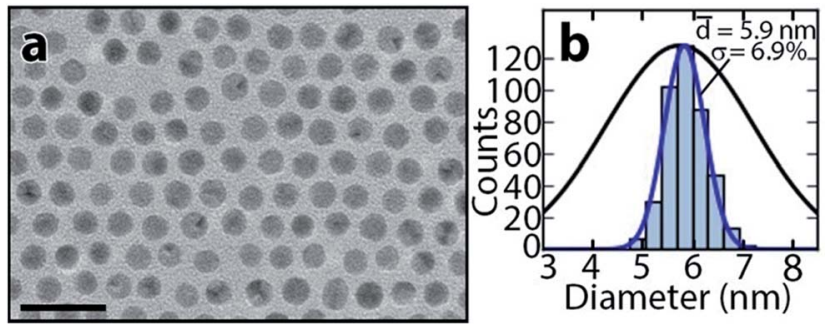

Fig. 3 (a) TEM image of Au NPs obtained in SSP by collecting the resulting precipitate from increasing the toluene: DDA/EtOH ratio from $5: 5$ to $5: 6$ with a $20 \mathrm{~nm}$ scale bar shown; (b) Gaussian fit and average NP size of NPs in (a), as well as an overlaying Gaussian fit to the unprocessed Brust NPs.

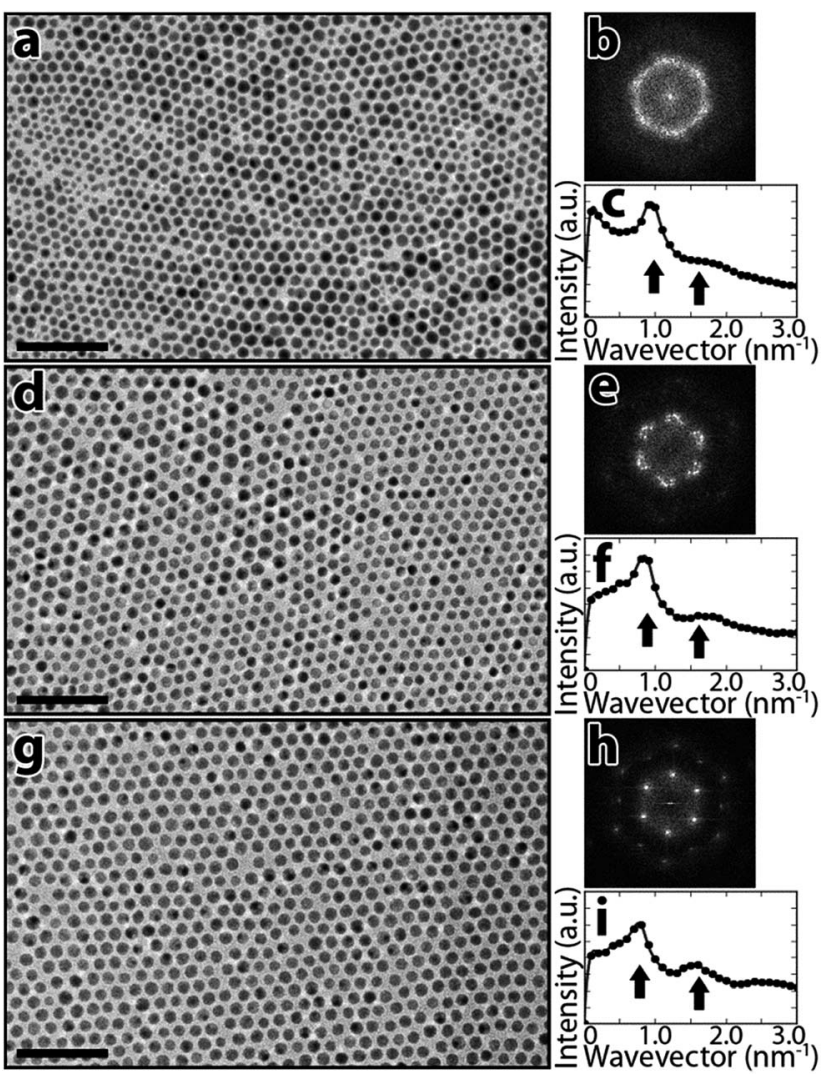

Fig. 4 (a) TEM image of a monolayer of Brust NPs formed using a Teflon ring apparatus; (b) 2D FFT of a larger $313 \mathrm{~nm} \times 313 \mathrm{~nm}$ region including the portion shown in (a); (c) radial average of (b); (d) - (f), (g)(i) analogous TEM images and FFT characterizations of NP monolayers with $\sigma=10 \%$ and $\sigma=6.9 \%$, respectively. Scale bars in (a), (d), (g) correspond to $50 \mathrm{~nm}$.

$\sigma=10 \%$ exhibits bright spots due a higher degree of hexagonally close-packed order. Dramatically sharper peaks as well as higher order peaks are seen in the FFT of the most monodisperse SSP-fractionalized sample $(\sigma=6.9 \%)$, indicating a high degree of order within the monolayer. These results are confirmed by the radial average of the 2D FFT (see arrows in Fig. 4i). A second peak in the radial FFT is observed at $\sim 1.5 \mathrm{~nm}^{-1}$ due to these higher order peaks in the 2D FFT. The second peak is either less pronounced or non-existent when using more polydisperse NPs (see Fig. 4(c and f)). These results show that NP size distributions with sub-10\% standard deviations are required for ordered hexagonal close-packing over several-hundred nm length scales.

We have demonstrated a simple, yet effective method for fractionalizing Au NPs synthesized via the Brust method into monodisperse fractions. The method is based on changing the NP ligand to DDA and using DDA/EtOH as a non-solvent. The effects of temperature, ligand type (notably ligand head group), and excess ligand concentration in the non-solvent on this system have been investigated. These results are significant because they shine a new light on phenomena at play in SSP and provide a simple, robust and tunable means for producing monodisperse Au NPs in organic solvents. 


\section{Conflicts of interest}

There are no conflicts to declare.

\section{Acknowledgements}

This work was supported by the Natural Sciences and Engineering Research Council of Canada and by MITACS.

\section{Notes and references}

1 Y. Ofir, B. Samanta and V. M. Rotello, Chem. Soc. Rev., 2008, 37, 1745.

2 S. Sarina, E. R. Waclawik and H. Zhu, Green Chem., 2013, 15, 1814.

3 X. Li, J. Zhu and B. Wei, Chem. Soc. Rev., 2016, 45, 3145.

4 Y.-C. Yeh, B. Creran and V. M. Rotello, Nanoscale, 2012, 4, 1871.

5 X. Zhang, Cell Biochem. Biophys., 2015, 72, 771.

6 Z. Wang and L. Ma, Coord. Chem. Rev., 2009, 253, 1607.

7 C. P. Shaw, D. G. Fernig and R. Levy, J. Mater. Chem., 2011, 21, 12181.

8 M. B. Ross, C. A. Mirkin and G. C. Schatz, J. Phys. Chem. C, 2016, 120, 816.

9 J. Van Rie and W. Thielemans, Nanoscale, 2017, 9, 8525.

10 P. Zhao, N. Li and D. Astruc, Coord. Chem. Rev., 2013, 257, 638.
11 H. Hiramatsu and F. E. Osterloh, Chem. Mater., 2004, 16, 2509.

12 X. Ji, X. Song, J. Li, Y. Bai, W. Yang and X. Peng, J. Am. Chem. Soc., 2007, 129, 13939.

13 N. Zheng, J. Fan and G. D. Stucky, J. Am. Chem. Soc., 2006, 128, 6550.

14 Y. Mori, KONA Powder Part. J., 2015, 32, 102.

15 B. Kowalczyk, I. Lagzi and B. A. Grzybowski, Curr. Opin. Colloid Interface Sci., 2011, 16, 135.

16 P. S. Vengsarkar and C. B. Roberts, J. Phys. Chem. C, 2013, 117, 14362.

17 C. B. Murray, D. J. Norris and M. G. Bawendi, J. Am. Chem. Soc., 1993, 115, 8706.

18 A. Taleb, C. Petit and M. P. Pileni, Chem. Mater., 1997, 9, 950.

19 N. Fishelson, I. Shkrob, O. Lev, J. Gun and A. D. Modestov, Langmuir, 2001, 17, 403.

20 M. Brust, M. Walker, D. Bethell, D. J. Schiffrin and R. Whyman, J. Chem. Soc., Chem. Commun., 1994, 801.

21 M. Brust, D. Bethell, D. J. Schiffrin and C. J. Kiely, Adv. Mater., 1995, 7, 795.

22 S. A. Tovstun and V. F. Razumov, J. Nanopart. Res., 2017, 19, 8.

23 S. Gravelsins, M. Hasham, Y. Lin, K. Yu, M. Tie, C. Goh and A.-A. Dhirani, Soft Matter, 2017, 13, 2437.

24 V. Santhanam, J. Liu, R. Agarwal and R. P. Andres, Langmuir, 2003, 19, 7881. 